\title{
Echinacoside Improves Cognitive Impairment by Inhibiting A $\beta$ Deposition Through the PI3K/AKT/Nrf2/PPARY Signaling Pathways in APP/PS1 Mice
}

hui qiu

China medical university

min xue liu ( $\square$ liuxuemin@cmu.edu.cn )

China Medical University Second Hospital: Shengjing Hospital of China Medical University https://orcid.org/0000-0002-1072-573X

\section{Research Article}

Keywords: Alzheimer's disease, Echinacoside, Nuclear factor erythroid 2-related factor 2, BACE1, $\beta$ amyloid protein

Posted Date: December 28th, 2021

DOI: https://doi.org/10.21203/rs.3.rs-1158047/v1

License: (c) (1) This work is licensed under a Creative Commons Attribution 4.0 International License.

Read Full License 


\section{Abstract}

Echinacoside $(\mathrm{ECH})$, a phenylethanoid glycoside, has protective activity in neurodegenerative disease, including anti-inflammation and antioxidation. However, the effects of ECH in Alzheimer's disease (AD) are not very clear. This present study investigates the role and mechanism of ECH in the pathological process of AD. APP/PS1 mice were treated with $\mathrm{ECH}$ in $50 \mathrm{mg} / \mathrm{kg} / \mathrm{d}$ for 3 months. Morris water maze, nesting test and immunofluorescence staining were used to observe whether $\mathrm{ECH}$ could improve $\mathrm{AD}$ pathology. Western blot was used to study the mechanism of ECH improving AD pathology. The results showed that ECH alleviated the memory impairment of APP/PS1 mice by reducing the time of escape latency as well as increasing the times of crossing the platform and rescued the impaired ability to construct nests. In addition, ECH significantly reduced the deposition of senile plaques in the brain and decreased the expression of BACE1 in APP/PS1 mice through activating PI3K/AKT/ Nrf2/PPARY pathway. Furthermore, ECH decreased ROS formation, GP91 and 8-OHdG expression, upregulated the expression of SOD1 and SOD2 as well as activating the PI3K/AKT/Nrf2 signaling pathway. Moreover, ECH inhibited glia cells activation, pro-inflammatory cytokine IL-1 $\beta$ and TNF-a release, NLRP3 inflammasome formation through TXNIP/Trx-1 signaling pathway. In conclusion, this paper reported that ECH improved cognitive function, inhibited oxidative stress and inflammatory response in AD. Therefore, we suggest that $\mathrm{ECH}$ may be considered as a potential drug for $\mathrm{AD}$ treatment.

\section{Introduction}

Alzheimer's disease (AD) is the most common progressive and devastating disease of the elderly that is related to cognition impairment [1]. The pathological features of $A D$ include amyloid beta $(A \beta)$ deposition and intracellular neurofibrillary tangles (NFT) containing hyperphosphorylated tau protein, as well as loss of synapses and neurons [2]. A $\beta$ derived from amyloid precursor protein (APP) by sequential proteolytic cleavages via $\beta$-secretase (BACE1) and $\gamma$-secretase [3]. BACE1 is a rate-limiting enzyme in the production of $A \beta$ and inhibiting the activity of BACE1 appears to be a prime target for improving AD pathogenesis [4]. Peroxisome proliferator-activated receptor-y (PPARY) is a transcription factor that regulates the activity of the BACE1 promoter [4]. A previous study indicated that activating PPARy could reduce the generation of $A \beta$ and the mechanism related to inhibite BACE1 expression [5]. These studies indicate that PPARY may be involved in production of $A \beta$.

In addition, oxidative stress and inflammation play essential roles in AD pathogenesis [6-7]. Oxidative stress participates in $A D$ development by increasing $A \beta$ generation and tau hyperphosphorylation [6], suggesting that antioxidants may be a potential therapy for AD. Nuclear factor erythroid 2-related factor 2 (Nrf2), a transcription factor that regulates the antioxidant and anti-inflammatory response (Ren et al. 2020), related to AD-mediated cognitive decline [8]. Previous study showed that the expression of Nrf2 was decreased in AD brains [9] and Nrf2 reduction exacerbated cognitive deficits in a mouse model of $A D$ [8]. Activating Nrf2 protects against detrimental stress by promoting the antioxidative defense pathway and ameliorates cognitive impairment in the AD model mouse [10-12]. Therefore, Nrf2 has emerged as a new therapeutic target in AD. 
Echinacoside $(\mathrm{ECH})$ is a natural phenylethanoid glycoside that derived from Echinacea angustifolia DC [13]. It performs numerous pharmacological activities, including antioxidant and anti-inflammation, combined with neuroprotective effects [14]. Recent studies have shown that ECH has protective effects on MPTP/MPP ${ }^{+}$-induced neurotoxicity in the mouse model of Parkinson's disease (PD) by inhibiting inflammatory response and regulating the autophagy pathway $[7,15]$. It also plays a protective role in other PD models [16]. Furthermore, ECH extends the lifespan of Caenorhabditis elegans by increasing resistance to oxidative stress and protecting from A $\beta$-induced toxicity [17]. More importantly, ECH ameliorates the memory impairment and cholinergic deficit induced by $A \beta$ [18]. These studies indicated that $\mathrm{ECH}$ had the potency to prevent $A D$ progression. However, the possible therapeutic target of $E C H$ in $A D$ is not clear. Thus, the aims of this study were to testify the effect of ECH on AD pathology in APP/PS1 mice. We demonstrated that long-term treatment with $\mathrm{ECH}$ improved the cognitive impairment of APP/PS1 mice by decreasing A $\beta$ production, oxidative stress and inflammation responses.

\section{Materials And Methods}

\section{Animals and treatment}

The APPswe/PSEN1dE9 (APP/PS1) transgenic mice, a C57BL6 strain of mice with human APPSwe and PS1-dE9 mutations, were purchased from the Jackson Laboratory and maintained them under standard conditions (room temperature of $22-25^{\circ} \mathrm{C}$ and $12 \mathrm{~h}$ light/dark cycle). Genotyping was performed by PCR analysis of tail DNA. 5-month-old male mice were randomly divided into two groups ( $n=6$ per group); vehicle and $\mathrm{ECH}$ group (50 mg/kg/d). All groups of mice were kept under the same conditions for 3 months before behavior tests were performed. All experimental procedures performed using animals were approved by the Laboratory of Animal Ethical Committee of China Medical University (CMU2020397).

\section{Behavior Tests}

After 3 months of treatment, behavior tests were performed using the Morris water maze and nest building tests. Two days before the test, mice were trained three times per day for two consecutive days with a visible platform. For the next 5 days, a navigation test was carried out. First, the platform was hidden, and both the latency time of mice finding the platform were recorded using the water maze system (ZH0065; Zhenghua Bioequipment, China). In the probe trial, the platform was removed, and the number of times that mice crossed the platform was recorded.

In the nest building test, mice were individually placed in a cage and randomly placed with eight square pieces of paper $(5 \times 5 \mathrm{~cm})$. The change in the pieces of paper was observed for 7 days and photographically recorded. The nest-building abilities were assessed according to the scoring criteria: $1=$ no biting/tearing, with random dispersion of the paper; $2=$ no biting/tearing of paper, with gathering in a corner/side of the cage; 3 = moderate biting/tearing of paper, with gathering in a corner/side of the cage; and 4 = extensive biting/tearing of paper, with gathering in a corner/side of the cage. 
At the end of the behavior tests, the mice were anesthetized and perfused with PBS. After that, the brains were quickly collected on ice, and one hemisphere was frozen and stored at $-80^{\circ} \mathrm{C}$, and the other was immersion-fixed in $4 \%$ paraformaldehyde for the histological study.

\section{Measurement of superoxide dismutase (SOD) activity and reactive oxygen species (ROS)}

The cerebral cortex was homogenized and centrifuged at $4^{\circ} \mathrm{C}$. The supernatant was collected to assay for protein concentration by BCA protein assay kit. After the protein concentration was measured, the activity of SOD was determined by SOD assay kit according to the manufacturer's instructions (Najing Jiancheng A001-3-2). Cell suspension of fresh tissues was incubated with DCFH-DA (Najing Jiancheng E004-1-1) for $30 \mathrm{~min}$ at $37^{\circ} \mathrm{C}$ and the fluorescence intensity of ROS was detected by enzyme labeling instrument.

\section{Immunofluorescence staining}

The frozen sections were blocked with $5 \%$ goat serum for 30 min and incubated with anti-A $\beta$ antibody (mouse monoclonal; 1:100, Santa Cruz) or anti-A $\beta$ antibody /anti-GFAP antibody (rabbit polyclonal; 1:200, Cell signal)/anti-lba-1 (rabbit polyclonal; 1:200, Wako) overnight at $4^{\circ} \mathrm{C}$. The sections were washed with PBS and then incubated with Alexa Fluor 488- or Alex Fluor 594-conjugated secondary antibodies for 2 h. Images were acquired using the laser scanning confocal microscope (TCS SP8, Leica, Germany).

\section{Immunohistochemistry}

Paraffin-embedded brains were sectioned at a thickness of $5 \mu \mathrm{m}$. A series of three equally spaced brain sections were used for this experiment. After the blockage of goat serum for $30 \mathrm{~min}$, the sections were incubated with rabbit anti-Nrf2 (rabbit polyclonal; 1:1000, Abcam) overnight at $4^{\circ} \mathrm{C}$. Next, sections were treated with appropriate secondary antibodies for $1 \mathrm{~h}$ and third antibody for $30 \mathrm{~min}$ at room temperature, and subsequently developed in DAB for $3 \mathrm{~min}$. Finally, the sections were dehydrated and sealed. The images of half mouse brains were obtained from a light microscope (DM4000B, Leica, Germany).

\section{Quantitative real-time polymerase chain reaction (RT-PCR)}

Total RNA of mouse tissues was extracted using Total RNA Kit according to the manufacturer's instruction and reverse transcribed into CDNA. The cDNA synthesis conditions were $37^{\circ} \mathrm{C}$ for $15 \mathrm{~min}$ and then $85^{\circ} \mathrm{C}$ for $5 \mathrm{~s}$. Quantitative real-time PCR was performed in the 7300 Sequence Detection System using the SYBR Green PCR Master mix (AG11702, Accurate Biology, China). At least three independent assays of each cDNA sample were conducted. The primers and probes used in PCR are listed:

TNF-a:

F-AGCCCCCAGTCTGTATCCTT,

R-ACAGTCCAGGTCACTGTCCC;

IL-1 $\beta$ : 
F-AGCCAAGCTTCCTTGTGCAAGTGT,

R-GCTCTCATCAGGACAGCCCAGGT;

BACE1:

F-GGAACCCATCTCGGCATCC

R-TCCGATTCCTCGTCGGTCTC

GAPDH:

F-TGCAGTGGCAAAGTGGAGAT,

R-TTTGCCGTGAGTGGAGTCATA.

The gene expression values were normalized to those of GAPDH.

\section{Western blot}

Mouse tissues were lysed in RIPA lysis buffer on ice for $3 \mathrm{~h}$ and then were centrifuged at $12000 \mathrm{~g}$ for 15 min at $4^{\circ} \mathrm{C}$ to collect the supernatant. The protein concentrations were measured using the Bradford assay kit. Protein, $30 \mu \mathrm{g}$ per lane, was separated by $10 \%$ SDS-PAGE gel and then transferred to PVDF membranes. Membranes were blocked with $5 \%$ nonfat milk for 30 min and incubated with primary antibodies for ADAM10 (rabbit polyclonal; 1:1000, Abcam), BACE1 (rabbit polyclonal; 1:1000, Abcam), PS1 (rabbit polyclonal; 1:1000, Cell signal), PEN2 (rabbit polyclonal; 1:1000, Cell signal), Nicastrin (NCT) (rabbit polyclonal; 1:1000, Cell signal), APH-1 (rabbit polyclonal; 1:1000, Thermo), Nrf2 (rabbit polyclonal; 1:1000, Abcam), HO-1 (rabbit polyclonal; 1:1000, Abcam), GP91 (rabbit polyclonal; 1:1000, Abcam), SOD1 (rabbit polyclonal; 1:1000, Proteintech), SOD2 (rabbit polyclonal; 1:1000, Proteintech), TXNIP (rabbit polyclonal; 1:1000, Abcam), NLRP3 (rabbit polyclonal; 1:1000, Abcam), Trx-1 (rabbit polyclonal; 1:1000, Abcam), p-AKT (rabbit polyclonal; 1:1000, Cell signal), AKT (rabbit polyclonal; 1:1000, Cell signal), p-PI3K (rabbit polyclonal; 1:1000, Cell signal), PI3K (rabbit polyclonal; 1:1000, Cell signal), GAPDH (rabbit polyclonal; 1:10000, Cell signal), p-PPARy (rabbit polyclonal; 1:1000, Cell signal), and PPARy (rabbit polyclonal; $1: 1000$, Cell signal) overnight at $4^{\circ} \mathrm{C}$. After washing three times for $15 \mathrm{~min}$, the membranes were incubated with HRP-conjugated secondary antibody for $1.5 \mathrm{~h}$ and detected using ECL.

\section{Statistical analysis}

All data are represented as mean \pm standard error of the mean (SEM). Statistical significances between the $\mathrm{ECH}$ treatment group and the vehicle control treatment group were determined by $\mathrm{t}$-test. The difference was considered to be statistically significant when $P<0.05$.

\section{Results}




\section{ECH improved memory deficits in APP/PS1 mice}

To address whether ECH could improve memory impairments in APP/PS1 mice, we tested the mice using the Morris water maze and nest building tests (Figure 1), which assessed spatial learning and memory function. The results showed that the latency time of mice finding the platform were significantly reduced in ECH treatment groups on the navigation test. The platform crossing times were significantly higher in the probe trial compared with the vehicle group (Figure $1 \mathrm{~A}-\mathrm{E}$ ). Moreover, ECH could improve the ability of nest construction compared with the vehicle group (Figure $1 \mathrm{~F}-\mathrm{G}$ ). The results suggest that $\mathrm{ECH}$ can significantly improve learning and memory dysfunction in APP/PS1 mice.

\section{ECH inhibited the production of $A \beta_{1-42}$ production as detected by immunofluorescence staining}

To determine the mechanism of ECH to improve the learning and memory abilities of mice, senile plaque deposition was detected by immunofluorescence staining. The results showed that the number of $A \beta_{1-42}$ in the cortex and hippocampus of mice decreased significantly compared with the vehicle group (Figure 2), indicated that $E C H$ alleviated cognitive impairment by reducing the production of $A \beta_{1-42}$.

\section{ECH reduced BACE1 expression in the APP/PS1 mice}

$A \beta$ is produced by the APP through the amyloid cleavage pathway, while the APP non-amyloid pathway can inhibit the production of $A \beta$. Therefore, the amyloid pathway cleavage enzyme BACE1 and nonamyloid cleavage enzyme ADAM10, as well as their production of cleavage were assessed. The results showed that there was a significant decrease in the expression of BACE1 and production of cleavage sAPP $\beta$ after ECH treatment (Fig 3 A, D, E, F). However, the expression of ADAM10, sAPPa and $\gamma$-secretase (PS1, NCT, PEN2 and APH-1) were not significantly different compared with the vehicle group (Figure 3 A$\mathrm{C}, \mathrm{G}-\mathrm{K})$. These results suggested that ECH administration could down-regulate the level of BACE1 to inhibit $A \beta$ generation.

\section{ECH significantly inhibited oxidative stress}

Oxidative stress plays a vital role in $A D$ pathogenesis and promotes $A D$ development via increasing $A \beta$ deposition and tau hyperphosphorylation [7]. Therefore, antioxidant drugs may have a therapeutic effect on AD. We measured ROS and antioxidant enzyme activity to investigate the relationship between ECH and oxidative stress. After ECH treatment, the content of ROS significantly decreased, while antioxidant enzyme (SOD1 and SOD2) activity increased significantly (Figure 4 A-D, F).

Oxidative stress-induced damage occurs to the lipids of cellular membranes, proteins, and DNA [19]. 8hydroxy-2'-deoxyguanosine (8-OHdG), one of the primary forms of free radical-induced oxidative damage, has been recognized as a biomarker of oxidative stress [19]. Moreover, GP91 has also been recognized as biomarkers of oxidative stress [20]. In this study, the levels of GP91 and 8-OHdG were significantly decreased after ECH treatment (Figure $4 \mathrm{~A}, \mathrm{E}, \mathrm{G}$ ). Taken together, these results illustrated that ECH could 
significantly reduce oxidative stress, and its mechanism might be related to increase the activity of antioxidant enzymes.

\section{ECH restrained glia cells activation and pro-inflammatory cytokine release}

ECH alleviates LPS-induced cell apoptosis and inflammation in rat intestinal epithelial cells [21] and possesses a neuroprotective effect via inhibiting inflammation. However, it is unclear how ECH improves $A D$ pathology by anti-inflammation in the APP/PS1 mice.

GFAP or Iba- 1 and A $\beta$ were stained with double-labeled immunofluorescence, and we found that $\mathrm{ECH}$ could significantly inhibit the activation of glial cells around senile plaques (Figure 5 A-D). Western blotting results showed that ECH inhibited the expression of GFAP and Iba-1 compared with the vehicle group (Figure $5 \mathrm{E}$ ).

To examine whether ECH inhibits inflammatory responses in APP/PS1 mice, pro-inflammatory cytokines such as TNF- $\alpha$ and IL-1 $\beta$ were detected by ELISA and RT-PCR (Figure 5 F-I). The results suggest that ECH inhibited the expression of TNF- $\alpha$ and IL-1 $\beta$ to decrease the inflammation response.

\section{ECH activated PI3K/AKT/ Nrf2/PPARY signaling pathways}

Transcription factor PPARy regulates the activity of the BACE1 promoter, and activating PPARY can inhibit BACE1 $[5,16]$. As an upstream signal molecule of PPARy, deficiency of Nrf2 attenuated PPARY transcriptional activity [22]. Previous study indicated that PI3K/AKT signaling pathway was participated in activating Nrf2 [23]. In our study, PI3K, AKT, Nrf2, PPARY and HO-1 expression were assessed. The results showed that ECH increase the phosphorylation the levels of PI3K and AKT, promoted Nrf2 expression in the nucleus and cytoplasm, subsequently promote PPARY expression compared with the vehicle group (Fig $6 \mathrm{~A}-\mathrm{I}$ ). Moreover, ECH accelerated the expression of HO-1 (Figure 6 E-I). In conclusion, our results indicate that ECH improves AD pathology by activating PI3K/AKT/ Nrf2/PPAPY pathways.

\section{ECH inhibited NLRP3 inflammasome activation through TXNIP/Trx-1 signaling pathway}

Moreover, Nrf2 is related to inflammation by inhibiting NLRP3 inflammasome activation through TXNIP/Trx-1 complex regulation [11]. In our research, we found that the expression of NLRP3 inflammasome significantly decreased and Trx-1 expression increased after ECH treatment. However, the lever of TXNIP was down-regulated by ECH (Figure $6 \mathrm{~J}-\mathrm{M}$ ). Our results showed that ECH inhibited NLRP3 inflammasome activation by regulating TXNIP/Trx-1 pathway.

\section{Discussion}

In this study, we investigated the molecular mechanisms of ECH on AD using APP/PS1 mice. We found that $\mathrm{ECH}$ improves mouse cognitive function and reduces both senile plaque deposition and oxidative damage in the brain. 
Our data demonstrated that $\mathrm{ECH}$ ameliorated memory impairments and decreased $\mathrm{A} \beta$ generation in APP/PS1 mice. We found that ECH reduced $A \beta$ production by inhibiting the expression of BACE1, but there were no effects on $\mathrm{a}$ - or $\mathrm{Y}$-secretase. This finding is consistent with the research of Dai et al [24].

BACE1 expression is regulated by a variety of transcription factors, including NF-KB and PPARY [4]. It has been reported that the level of PPARY significantly reduced in the brains of $A D$ patients and mice models [25]. PPARY depletion enhances BACE1 mRNA levels by promoting BACE1 gene promoter activity. Conversely, overexpression of PPARY or PPARy activators, reduced BACE1 gene promoter activity and inhibited BACE1 expression [26], which ultimately reduces the generation of $A \beta$ [5]. Although our results showed that ECH significantly inhibited the expression of BACE1, it was unclear whether the mechanism of ECH is involving in activating PPARy. In our study, we found that ECH can activate PPARy, leading to the down-regulation of BACE1 and the inhibition of AB. Furthermore, PPARY as a direct downstream transcriptional target of Nrf2, its promoter activity can be activated by Nrf2. On the contrary, Nrf2 deficiency also leads to decreased expression of PPARy [22]. A decline in the expression of the transcription factor Nrf2 have been observed in AD brains and Nrf2 induction ameliorates cognitive impairment in the AD model mouse [12, 27]. To assess whether ECH activate PPARy by promoting Nrf2 expression, thereby protecting AD model mice against disease progression, we detected Nrf2 expression in the nucleus and cytoplasm. The results showed that ECH promoted Nrf2 translocation to the nucleus and the expression of downstream genes PPARY. A previous study showed that PI3K/AKT pathway is related to Nrf2 activation [23]. After treatment with the PI3K/AKT inhibitor Ly294002, the p-AKT and Nrf2 levels, as well as the Nrf2 nuclear translocation, were significantly suppressed [23]. Anthocyanins (potent antioxidant and neuroprotective agents) reduce $A \beta$ oligomer-induced neurotoxicity by the PI3K/AKT/Nrf2 pathway [28]. Panax notoginseng saponins activated Nrf2 in a PI3K/AKT pathway-dependent manner and protected against barrier disruption [29]. In this study, we determined whether ECH could increase Nrf2 nuclear translocation by activating the PI3K/AKT pathway. Our results show that ECH up regulates the phosphorylation level of PI3K and then induces AKT to activate Nrf2. The activated Nrf2 can increase the promoter activity of PPARY and inhibit the expression of BACE1.

Accumulating evidence indicates that $A D$ is related to oxidative stress [30]. Our study found that the antioxidant enzyme activity of SOD1 and SOD2 increased after ECH treatment, and SOD1 expression was upregulated. The levels of the oxidative stress biomarkers (GP91 and 8-OHdG) were significantly decreased after ECH treatment. Furthermore, ECH inhibited glia cells activities and then reduced the release of inflammatory cytokines, including IL-1 $\beta$ and TNF-a. Nrf2 acted as the key transcriptional regulator [31], against oxidative stress damage in $A D$ [25] by increasing the endogenous antioxidant capacity [32]. Recently, studies have shown that Nrf2 induction increased GSH levels and attenuated reactive astrocytosis [33]. Resveratrol attenuates oxidative stress by PI3K/AKT-induced Nrf2 activation [34]. Panax notoginseng saponins activated antioxidant signaling by Nrf2 in a PI3K/AKT pathwaydependent manner and protected against barrier disruption [29]. Our results indicated that ECH inhibited oxidative stress damage by PI3K/AKT-induced Nrf2 activation pathway. 
In addition, chronic inflammation has a vital role in the onset and progression of AD [35]. Nrf2, thioredoxin interacting protein (TXNIP) and nucleotide-binding oligomerization domain-like receptor protein 3 (NLRP3) inflammasome pathways are closely related to inflammation-related diseases [36]. The NLRP3 inflammasome is a complex of multi-proteins that regulate inflammation by activating the secretion of the pro-inflammatory cytokine [37]. TXNIP as an endogenous regulator can interact with NLRP3, which activates the NLRP3 inflammasome and promotes inflammatory responses [38]. A previous study showed that Nrf2 inhibits NLRP3 inflammasome activation by regulating the TXNIP/Trx-1 complex [39]. DI-3-n-butylphthalide treatment suppresses TXNIP-NLRP3 interaction and inhibits NLRP3 inflammasome activation by upregulating Nrf2 [11]. Our results suggested that ECH decreased the TXNIP level and inhibited NLRP3 inflammasome activation against the neuroinflammation associated with Nrf2.

\section{Conclusion}

$\mathrm{ECH}$ decreased the $A \beta$ deposition by reducing the expression of BACE1 through activating $\mathrm{PI3K} / \mathrm{AKT}$ /Nrf2/PPARy signaling pathway. Furthermore, ECH promoted Nrf2 via the PI3K/AKT signaling pathway against oxidative stress, inhibited TXNIP-NLRP3 interaction, reduced NLRP3 inflammasome against inflammation, and improved AD pathology (Fig 7).

\section{Declarations}

\section{Data Availability}

The datasets used and/or analyzed during the current study are available from the corresponding author on reasonable request.

\section{Conflicts of Interest}

The authors declare that they have no competing interests.

\section{Authors Contributions}

H.Q., performed most of the experiments and analysed the data; X.M.L., designed and wrote manuscript. All authors have read and approved the final manuscript.

\section{Funding}

This work was supported by the Science and Technology Foundation of Liaoning Educational Committee (L2014290).

Consent to Participate Not applicable

Consent to Publication Not applicable

Acknowledgements We thanks Shen-Shen Yao and Jia-Ren Zhou for technical assistance. 


\section{References}

1. Singh A, Agarwal S, Singh S (2020) Age related neurodegenerative Alzheimer's disease: Usage of traditional herbs in therapeutics. Neuroscience letters 717:134679. doi:10.1016/j.neulet.2019.134679

2. Ozben T, Ozben S (2019) Neuro-inflammation and anti-inflammatory treatment options for Alzheimer's disease. Clinical biochemistry 72:87-89. doi:10.1016/j.clinbiochem.2019.04.001

3. Vassar R, Citron M (2000) Abeta-generating enzymes: recent advances in beta- and gamma-secretase research. Neuron 27 (3):419-422. doi:10.1016/s0896-6273(00)00051-9

4. Lin N, Chen LM, Pan XD, Zhu YG, Zhang J, Shi YQ, Chen XC (2016) Tripchlorolide Attenuates $\beta$-amyloid Generation via Suppressing PPARY-Regulated BACE1 Activity in N2a/APP695 Cells. Mol Neurobiol 53 (9):6397-6406. doi:10.1007/s12035-015-9542-2

5. Cao G, Su P, Zhang S, Guo L, Zhang H, Liang Y, Qin C, Zhang W (2016) Ginsenoside Re reduces A $\beta$ production by activating PPARy to inhibit BACE1 in N2a/APP695 cells. Eur J Pharmacol 793:101-108. doi:10.1016/j.ejphar.2016.11.006

6. Chen Z, Zhong C (2014) Oxidative stress in Alzheimer's disease. Neurosci Bull 30 (2):271-281. doi:10.1007/s12264-013-1423-y

7. Zhu S, Wang J, Zhang Y, He J, Kong J, Wang JF, Li XM (2017) The role of neuroinflammation and amyloid in cognitive impairment in an APP/PS1 transgenic mouse model of Alzheimer's disease. CNS Neurosci Ther 23 (4):310-320. doi:10.1111/cns.12677

8. Branca C, Ferreira E, Nguyen TV, Doyle K, Caccamo A, Oddo S (2017) Genetic reduction of Nrf2 exacerbates cognitive deficits in a mouse model of Alzheimer's disease. Hum Mol Genet 26 (24):48234835. doi:10.1093/hmg/ddx361

9. Ramsey CP, Glass CA, Montgomery MB, Lindl KA, Ritson GP, Chia LA, Hamilton RL, Chu CT, JordanSciutto KL (2007) Expression of Nrf2 in neurodegenerative diseases. J Neuropathol Exp Neurol 66 (1):7585. doi:10.1097/nen.0b013e31802d6da9

10. Bahn G, Jo DG (2019) Therapeutic Approaches to Alzheimer's Disease Through Modulation of NRF2. Neuromolecular Med 21 (1):1-11. doi:10.1007/s12017-018-08523-5

11. Wang CY, Xu Y, Wang X, Guo C, Wang T, Wang ZY (2019) DI-3-n-Butylphthalide Inhibits NLRP3 Inflammasome and Mitigates Alzheimer's-Like Pathology via Nrf2-TXNIP-TrX Axis. Antioxid Redox Signal 30 (11):1411-1431. doi:10.1089/ars.2017.7440

12. Qu Z, Sun J, Zhang W, Yu J, Zhuang C (2020) Transcription factor NRF2 as a promising therapeutic target for Alzheimer's disease. Free Radic Biol Med 159:87-102. doi:10.1016/j.freeradbiomed.2020.06.028 
13. Liu J, Yang L, Dong Y, Zhang B, Ma X (2018) Echinacoside, an Inestimable Natural Product in Treatment of Neurological and other Disorders. Molecules 23 (5). doi:10.3390/molecules23051213

14. Lei L, Yang F, Zhang T, Tu P, Wu L, Ito Y (2001) Preparative isolation and purification of acteoside and 2'-acetyl acteoside from Cistanches salsa (C.A. Mey.) G. Beck by high-speed counter-current chromatography. J Chromatogr A 912 (1):181-185. doi:10.1016/s0021-9673(01)00583-0

15. Liang Y, Chen C, Xia B, Wu W, Tang J, Chen Q, Tang L, Yang H, Zhang Z, Lu Y, Yang Y, Zhao Y (2019) Neuroprotective Effect of Echinacoside in Subacute Mouse Model of Parkinson's Disease. Biomed Res Int 2019:4379639. doi:10.1155/2019/4379639

16. Zhang Y, Long H, Zhou F, Zhu W, Ruan J, Zhao Y, Lu Y (2017) Echinacoside's nigrostriatal dopaminergic protection against 6-OHDA-Induced endoplasmic reticulum stress through reducing the accumulation of Seipin. J Cell Mol Med 21 (12):3761-3775. doi:10.1111/jcmm.13285

17. Chen W, Lin HR, Wei CM, Luo XH, Sun ML, Yang ZZ, Chen XY, Wang HB (2018) Echinacoside, a phenylethanoid glycoside from Cistanche deserticola, extends lifespan of Caenorhabditis elegans and protects from A $\beta$-induced toxicity. Biogerontology 19 (1):47-65. doi:10.1007/s10522-017-9738-0

18. Shiao YJ, Su MH, Lin HC, Wu CR (2017) Echinacoside ameliorates the memory impairment and cholinergic deficit induced by amyloid beta peptides via the inhibition of amyloid deposition and toxicology. Food Funct 8 (6):2283-2294. doi:10.1039/c7fo00267j

19. Valavanidis A, Vlachogianni T, Fiotakis C (2009) 8-hydroxy-2' -deoxyguanosine (8-OHdG): A critical biomarker of oxidative stress and carcinogenesis. J Environ Sci Health C Environ Carcinog Ecotoxicol Rev 27 (2):120-139. doi:10.1080/10590500902885684

20. Ma X, Wang F, Zhen X, Zhao L, Fang L, Dong Z, Chen W, Zhou X (2020) gp91(phox), a Novel Biomarker Evaluating Oxidative Stress, Is Elevated in Subclinical Hypothyroidism. Int J Endocrinol 2020:3161730. doi:10.1155/2020/3161730

21. Li L, Wan G, Han B, Zhang Z (2018) Echinacoside alleviated LPS-induced cell apoptosis and inflammation in rat intestine epithelial cells by inhibiting the mTOR/STAT3 pathway. Biomed Pharmacother 104:622-628. doi:10.1016/j.biopha.2018.05.072

22. Pi J, Leung L, Xue P, Wang W, Hou Y, Liu D, Yehuda-Shnaidman E, Lee C, Lau J, Kurtz TW, Chan JY (2010) Deficiency in the nuclear factor E2-related factor-2 transcription factor results in impaired adipogenesis and protects against diet-induced obesity. J Biol Chem 285 (12):9292-9300. doi:10.1074/jbc.M109.093955

23. Wen Z, Hou W, Wu W, Zhao Y, Dong X, Bai X, Peng L, Song L (2018) 6'-O-Galloylpaeoniflorin Attenuates Cerebral Ischemia Reperfusion-Induced Neuroinflammation and Oxidative Stress via PI3K/Akt/Nrf2 Activation. Oxid Med Cell Longev 2018:8678267. doi:10.1155/2018/8678267 
24. Dai Y, Han G, Xu S, Yuan Y, Zhao C, Ma T (2020) Echinacoside Suppresses Amyloidogenesis and Modulates F-actin Remodeling by Targeting the ER Stress Sensor PERK in a Mouse Model of Alzheimer's Disease. Front Cell Dev Biol 8:593659. doi:10.3389/fcell.2020.593659

25. Zhang M, Qian C, Zheng ZG, Qian F, Wang Y, Thu PM, Zhang X, Zhou Y, Tu L, Liu Q, Li HJ, Yang H, Li P, Xu X (2018) Jujuboside A promotes $A \beta$ clearance and ameliorates cognitive deficiency in Alzheimer's disease through activating Axl/HSP90/PPARy pathway. Theranostics 8 (15):4262-4278. doi:10.7150/thno.26164

26. Sastre M, Dewachter I, Rossner S, Bogdanovic N, Rosen E, Borghgraef P, Evert BO, Dumitrescu-Ozimek L, Thal DR, Landreth G, Walter J, Klockgether T, van Leuven F, Heneka MT (2006) Nonsteroidal antiinflammatory drugs repress beta-secretase gene promoter activity by the activation of PPARgamma. Proc Natl Acad Sci U S A 103 (2):443-448. doi:10.1073/pnas.0503839103

27. Osama A, Zhang J, Yao J, Yao X, Fang J (2020) Nrf2: a dark horse in Alzheimer's disease treatment. Ageing Res Rev 64:101206. doi:10.1016/j.arr.2020.101206

28. Ali T, Kim T, Rehman SU, Khan MS, Amin FU, Khan M, Ikram M, Kim MO (2018) Natural Dietary Supplementation of Anthocyanins via PI3K/Akt/Nrf2/HO-1 Pathways Mitigate Oxidative Stress, Neurodegeneration, and Memory Impairment in a Mouse Model of Alzheimer's Disease. Mol Neurobiol 55 (7):6076-6093. doi:10.1007/s12035-017-0798-6

29. Hu S, Wu Y, Zhao B, Hu H, Zhu B, Sun Z, Li P, Du S (2018) Panax notoginseng Saponins Protect Cerebral Microvascular Endothelial Cells against Oxygen-Glucose Deprivation/Reperfusion-Induced Barrier Dysfunction via Activation of PI3K/Akt/Nrf2 Antioxidant Signaling Pathway. Molecules 23 (11). doi:10.3390/molecules23112781

30. Ren P, Chen J, Li B, Zhang M, Yang B, Guo X, Chen Z, Cheng H, Wang P, Wang S, Wang N, Zhang G, Wu X, Ma D, Guan D, Zhao R (2020) Nrf2 Ablation Promotes Alzheimer's Disease-Like Pathology in APP/PS1 Transgenic Mice: The Role of Neuroinflammation and Oxidative Stress. Oxid Med Cell Longev 2020:3050971. doi:10.1155/2020/3050971

31. Johnson DA, Johnson JA (2015) Nrf2--a therapeutic target for the treatment of neurodegenerative diseases. Free Radic Biol Med 88 (Pt B):253-267. doi:10.1016/j.freeradbiomed.2015.07.147

32. Wei W, Lan XB, Liu N, Yang JM, Du J, Ma L, Zhang WJ, Niu JG, Sun T, Yu JQ (2019) Echinacoside Alleviates Hypoxic-Ischemic Brain Injury in Neonatal Rat by Enhancing Antioxidant Capacity and Inhibiting Apoptosis. Neurochem Res 44 (7):1582-1592. doi:10.1007/s11064-019-02782-9

33. Uruno A, Matsumaru D, Ryoke R, Saito R, Kadoguchi S, Saigusa D, Saito T, Saido TC, Kawashima R, Yamamoto M (2020) Nrf2 Suppresses Oxidative Stress and Inflammation in App Knock-In Alzheimer's Disease Model Mice. Mol Cell Biol 40 (6). doi:10.1128/mcb.00467-19 
34. Zhuang Y, Wu H, Wang X, He J, He S, Yin Y (2019) Resveratrol Attenuates Oxidative Stress-Induced Intestinal Barrier Injury through PI3K/Akt-Mediated Nrf2 Signaling Pathway. Oxid Med Cell Longev 2019:7591840. doi:10.1155/2019/7591840

35. Newcombe EA, Camats-Perna J, Silva ML, Valmas N, Huat TJ, Medeiros R (2018) Inflammation: the link between comorbidities, genetics, and Alzheimer's disease. J Neuroinflammation 15 (1):276. doi:10.1186/s12974-018-1313-3

36. Zhang Y, Gao Z, Gao X, Yuan Z, Ma T, Li G, Zhang X (2020) Tilianin Protects Diabetic Retina through the Modulation of Nrf2/TXNIP/NLRP3 Inflammasome Pathways. J Environ Pathol Toxicol Oncol 39 (1):89-99. doi:10.1615/JEnvironPatholToxicolOncol.2020032544

37. Li W, Cao T, Luo C, Cai J, Zhou X, Xiao X, Liu S (2020) Crosstalk between ER stress, NLRP3 inflammasome, and inflammation. Appl Microbiol Biotechnol 104 (14):6129-6140. doi:10.1007/s00253020-10614-y

38. Bharti V, Tan H, Zhou H, Wang JF (2019) Txnip mediates glucocorticoid-activated NLRP3 inflammatory signaling in mouse microglia. Neurochem Int 131:104564.

doi:10.1016/j.neuint.2019.104564

39. Hou Y, Wang Y, He Q, Li L, Xie H, Zhao Y, Zhao J (2018) Nrf2 inhibits NLRP3 inflammasome activation through regulating Trx1/TXNIP complex in cerebral ischemia reperfusion injury. Behav Brain Res 336:3239. doi:10.1016/j.bbr.2017.06.027

\section{Figures}

\section{Figure 1}

ECH treatment improved the cognitive capacity of APP/PS1 mice. APP/PS1 mice ( 5 months old) were treated with $\mathrm{ECH}$ (i.p., $50 \mathrm{mg} / \mathrm{kg} / \mathrm{d}$ ) for 3 months. Morris water maze tests with 2 days of visible platform training, 5 days of hidden platform testing, and a probe trial after $24 \mathrm{~h}$ of the last hidden platform test were used to evaluate cognitive ability. (A-B) Mice from different groups exhibited a similar escape latency to the visible platform on days 1-2, and the hidden platform tests showed that ECH administration decreased the escape latency from the 3rd-7th day. (C) A representative path showing the mouse performance in the hidden platform trail on the 5th day. (D-E) In the probe trial, ECH treatment significantly increased the times of crossing the platform's former location. Nest construction was visualized after Morris water maze tests. (F-G) ECH treatment significantly rescued the impaired ability to construct nests. Data were presented as the mean $\pm \mathrm{SEM} ; \mathrm{n}=6,{ }^{*} P<0.05 ; \star \star ~ P<0.01$. 
Figure 2

ECH treatment reduced senile plaque burden in APP/PS1 mice. Five-month-old APP/PS1 mice were treated with $\mathrm{ECH}$ for 3 months. (A) Immunofluorescent labeling of $A \beta$ showing the $A \beta$ plaque in the cortex and hippocampus of APP/PS1 mice. (B-C) Quantification of $A \beta$ fluorescence revealed a reduced number of $A \beta$ plaques in the cortex and hippocampus of APP/PS1 mice after $E C H$ treatment. Data were presented as the mean $\pm \mathrm{SEM} ; \mathrm{n}=6,{ }^{*} P<0.05 ; * \star P<0.01$.

\section{Figure 3}

ECH inhibited the activation of BACE1 and the secretion of SAPP $\beta$ in the cortex of APP/PS1 mice. Fivemonth-old APP/PS1 mice were treated with ECH for 3 months. (A-F) Immunoblot analysis showed the expression levels of ADAM10, BACE1, the secretion of SAPPa and SAPP $\beta$ in the cortex. (G-K) Immunoblot analysis reveals the expression level of the subunits of $y$-secretase, including PS1, NCT, PEN2 and APH-1.

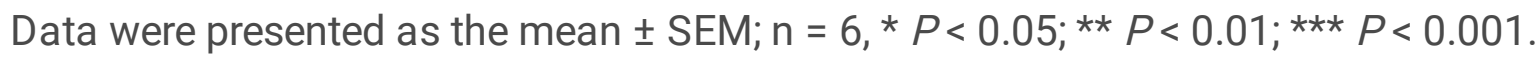

\section{Figure 4}

ECH treatment limited the oxidative stress in the cortex of APP/PS1 mice. Five-month-old APP/PS1 mice were treated with ECH for 3 months. (A, B, C, E) Immunoblot analysis showed the expression level of SOD1, SOD2, and GP91 in the cortex. (D) Changes in SOD activity in the cortex. (F) ROS production in the cortex was detected with the dichlorofluorescein diacetate probe. (G) Immunofluorescent labeling of 8OHdG showing the 8-OHdG in the cortex and hippocampus of APP/PS1 mice. Data were presented as the mean \pm SEM; $\mathrm{n}=6, * P<0.05 ;$ ** $P<0.01$.

\section{Figure 5}

ECH suppressed the neuroinflammatory reaction in the cortex and hippocampus of APP/PS1 mice. Fivemonth-old APP/PS1 mice were treated with $\mathrm{ECH}$ for 3 months. (A-D) ECH treatment suppressed the activation of microglia and astrocytes around the $A \beta$ plaque. (E) Immunoblot analysis showed the expression levels of GFAP and Iba-1. (F-G) ELISA showed that content of IL-1 $\beta$ and TNF- $a$ in the cortex. 
$(\mathrm{H}-\mathrm{I})$ The mRNA expression of IL-1 $\beta$ and TNF-a. Data were presented as the mean $\pm \mathrm{SEM} ; \mathrm{n}=6,{ }^{*} P<0.05$; ** $P<0.01$.

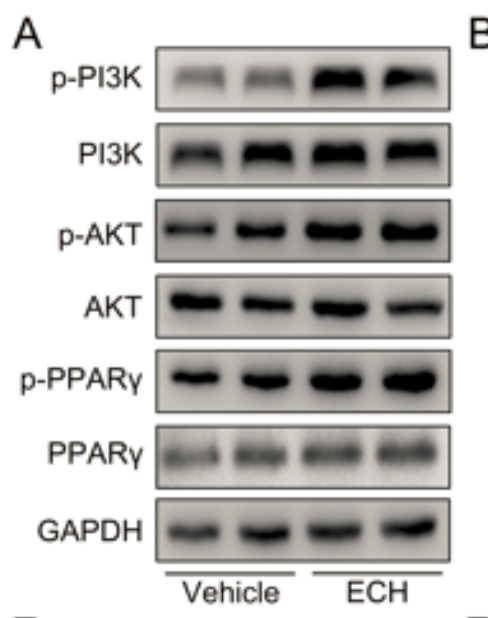

$\mathrm{E}$
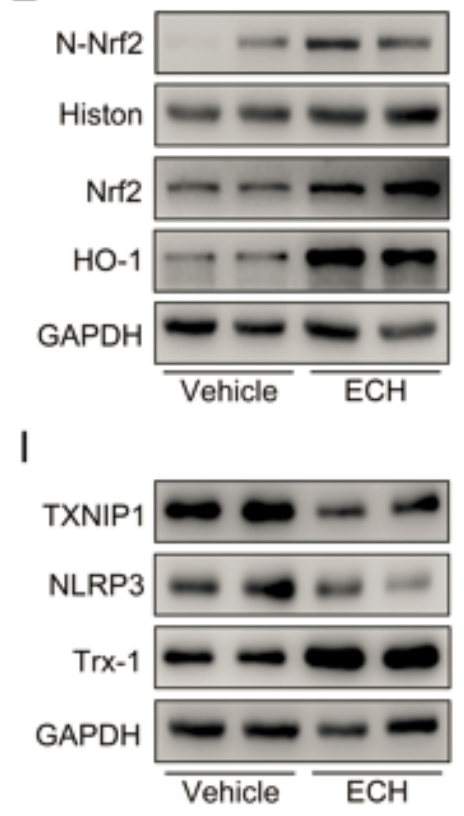

B

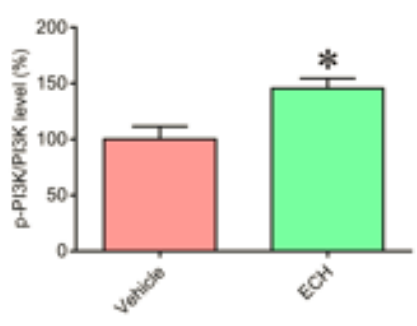

F

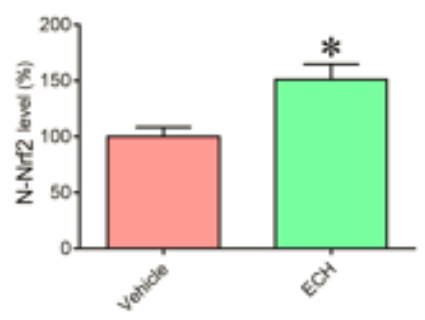

$J$

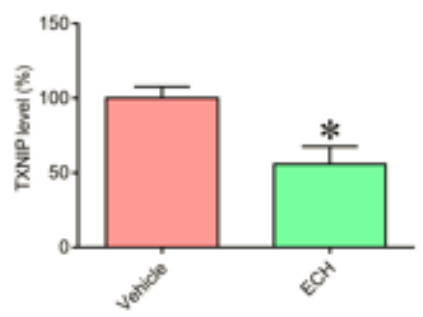

C

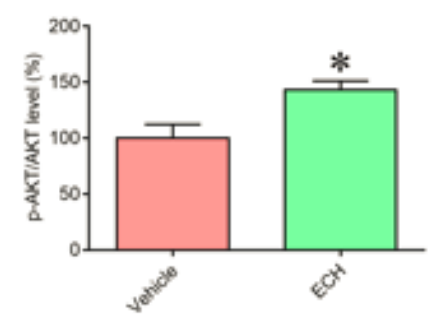

G

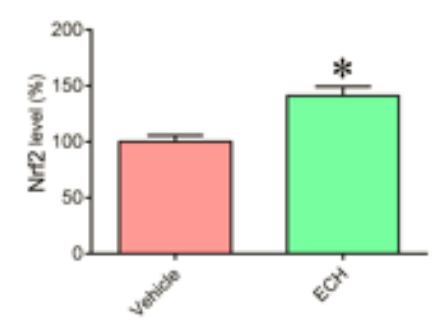

$\mathrm{K}$

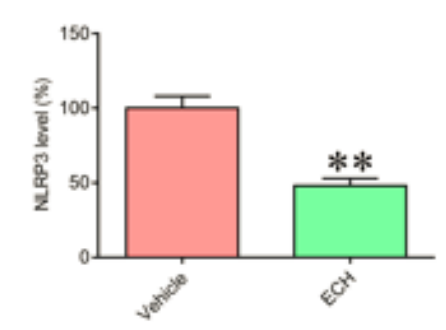

D

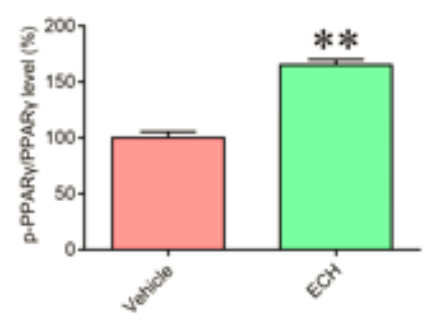

$\mathrm{H}$

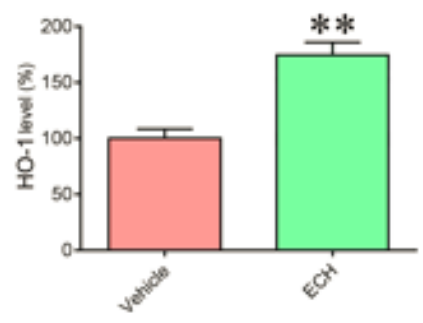

L

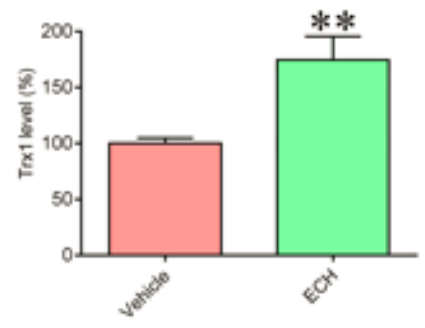

Figure 6

ECH activated the PI3K/AKT/Nrf2/PPARY signaling pathways in the cortex of APP/PS1 mice. Five-monthold APP/PS1 mice were treated with ECH for 3 months. (A-D) Immunoblot analysis showed the expression levels of p-PI3K, PI3K, p-AKT, AKT, p-PPARY and PPARY in the cortex. (E-H) Immunoblot analysis showed the expression level of Nucleu-Nrf2, Nrf2, and HO-1 in the cortex. (I) Immunostaining with anti-Nrf2 antibody showing the expression of Nrf2 in the cortex. (J-M) Western blot analysis showed the expression level of NLRP3, Trx-1 and TXNIP in the cortex. Data were presented as the mean \pm SEM; $n$ $=6, * P<0.05 ; * \star P<0.01$. 
Figure 7

Schematic diagram shows the mechanism of ECH ameliorated AD pathological process. $E C H$ inhibits BACE1 expression and oxidative stress by activating PI3K/AKT/Nrf2/PPARy signaling pathways, thus reducing $A \beta$ production. In addition, $E C H$ can reduce the production of $A \beta$ by inhibiting the release of inflammatory factors. 